\title{
Radiation-stimulated adsorption processes on the surface of beryllium oxide
}

\author{
Turgara T. Tusseyev*, Aktolkyn K. Danlybaeva, Saltanat S. Raimkul, \\ Aizhan A. Kiykabaeva, Ospan M. Doszhanov \\ Al-Farabi Kazakh National University, 050040 al-Farabi Ave., 71, Almaty, Kazakhstan \\ * Corresponding author: turgaratus@mail.ru \\ This article belongs to the Regular Issue. \\ (c) 2021, The Authors. This article is published in open access form under the terms and conditions of the Creative \\ Commons Attribution (CC BY) license (http://creativecommons.org/licenses/by/4.0/).
}

\section{Abstract}

The paper presents the study results of photo and gamma radiation effect on the beryllium oxide (BeO) surface properties. Photoadsorption studies of $\mathrm{O}_{2}$ on $\mathrm{BeO}$ by the methods of infrared (IR) spectroscopy and manometry with a change in the temperature of preliminary annealing from 473 to $1073 \mathrm{~K}$ show that samples subjected to preliminary training at $473 \mathrm{~K}$ are most active. The maximum of adsorption activity on $\gamma$-irradiated beryllium oxide is observed on the samples annealed at $673 \mathrm{~K}$ before the irradiation. The maximum of paramagnetic centers (PMCs) is also observed on samples annealed at this temperature. Comparison of electron paramagnetic resonance (EPR) and adsorption studies shows that absorption of $\mathrm{H}_{2}$ and $\mathrm{O}_{2}$ leads to the destruction of paramagnetic centers. It is assumed that, upon irradiation, adsorption centers with electron and hole modes are formed on the surface of $\mathrm{BeO}$.

\author{
Keywords \\ adsorption \\ irradiation \\ beryllium oxide \\ surface defects \\ spectra \\ temperature
}

Received: 19.11.2021

Revised: 18.01.2022

Accepted: 27.01.2022

Available online: 28.01.2022

\section{Introduction}

Beryllium oxides (BeO) [1] are widely used molecules in the nuclear and chemical industries [2, 3]. Recently, it was found that $\mathrm{BeO}$ functions as a catalyst with a developed surface, and its properties (catalytic) are influenced by external factors (e.g., temperature, radiation, etc.) [4].

It is now acceptable that nuclear radiation causes fundamental changes in the physical and chemical properties of materials. The above is largely true for the surface properties of oxide materials [5-11].

The changes in the adsorption properties of a solid body often occur due to radiation defects; thus, the interactions between adsorbates and adsorbents may be studied to determine the nature of these defects. By studying adsorption kinetics, the rate of radiation accumulation on the surface of irradiated materials can be traced to determine energy characteristics, e.g., depth of radiation level in the area and interaction energy with the adsorbate molecule. Thus, we can determine the mechanism for transferring energy from the irradiated solid to the adsorbed molecule, and so on [12, 13]. Using physical and chemical research methods (electron paramagnetic resonance (EPR), infrared (IR), and mass spectrometry), it is possible to investigate the nature of surface radiation defects, their adsorption activities, lifetime, and annealing activation energies. Adsorbates with pronounced acceptor (e.g.oxygen) and donor (e.g.hydrogen) properties may act as a "probe" as there will be an electron transfer between donor and acceptor.

Thus, the effects of radiation on solids with a developed surface and the impact of adsorption processes on irradiated materials are new research fields in electronic and structural factors. The present research makes it possible to understand the role of free and localized charge carriers in elementary acts of catalysis and chemisorption and the ability of adsorbed molecules to serve as traps for electrons and holes, as compared with other trappings of structural defects or impurity atoms located on the surface [14-17]. With sufficient information on these factors, it is possible to generate effective systems with predicted physical and technological properties [18]. Therefore, the aim of this work is to study the impact of irradiation on beryllium oxide properties.

\section{Experimental methods}

\subsection{Preparation of $\mathrm{BeO}$ surface}

In this research, beryllium oxide, the grade "for luminophores", was used to conduct the experiments the irradiation impact. This material had a form of white powder 
with a surface area of $60 \mathrm{~m}^{2} / \mathrm{g}$ and had impurities of other elements ( $\mathrm{Al}, \mathrm{Fe}, \mathrm{Mn}, \mathrm{Ca}, \mathrm{Si}$ ) from $10^{-2}$ to $10^{-4}$ weight $\%$. It is well-known that $\mathrm{BeO}$ is very hygroscopic [19], the weight loss during calcination at $900{ }^{\circ} \mathrm{C}$ may be up to $20 \%$ of the initial weight; thus, we tried not to overheat beryllium oxide samples.

\subsection{Characterization}

The surface properties were characterized using manometeric, infrared spectroscopic and radio spectroscopic methods. The apparatus, on which vacuum-temperature training of samples was carried out and gamma adsorption was studied, has two parallel pumping branches consisting of zeolite-coal adsorption pumps, diffusion pump type, and cooled traps with a heated oil-free high-vacuum valve. Preliminary studies had shown that there was no noticeable difference in the measurement results between the two pumping methods. Therefore, due to the advantage of the first pumping method consisting in the freedom from contamination by foreline pump oil vapors, we preferred to use it in the experiments.

Pressure measurements in the adsorbent-gas system in the gamma adsorption experiments were performed using a manometer pre-gauged for the gas used as adsorbate. The temperature in the system was measured using chromel-alumel, chromel-copper, and platinum-platinumrhodium thermocouples.

The investigated gases were mainly hydrogen and oxygen. Oxygen was obtained by decomposition of potassium permanganate at temperatures above $230{ }^{\circ} \mathrm{C}$ and further purified by passing it through a trap cooled with liquid nitrogen. Before obtaining the gas, the initial product was held for several hours at a temperature slightly lower than the starting reaction temperature. The electrolysis of distilled water produced hydrogen, which was purified further by diffusion through heated palladium capillary walls and then passed through a trap cooled with liquid nitrogen. The resulting gas was stored in the gas production system for 3-4 $\mathrm{h}$ before being pumped out and produced again to avoid contamination. Although the gases were thoroughly purified, there were still impurities in their composition. Mass spectrometric analysis showed that oxygen contains carbon dioxide and hydrogen has water vapor.

The gamma unit with a dose rate in the central channel of $200 \mathrm{rad} / \mathrm{s}$ was used as a source of $\gamma$-quanta, with the radiation source being the preparation of the radioactive isotope ${ }^{6 \circ} \mathrm{Co}$. The dose was determined using a ferrous sulfate dosimeter. The radiation dose was calculated according to the formula:

$$
D=k_{\gamma} M t / R^{2},
$$

where $R$ is the distance in meter; $t$ is time in h; $M$ is isotope activity in millicuries; $k_{\gamma}$ is the gamma constant, indicating the dose rate of a point source. The following for- mula was used to calculate the amount of gammaadsorbed gas:

$$
N=3 \cdot 3 \cdot 10^{16} \Delta p V,
$$

where $N$ is the amount of adsorbed gas in $\mathrm{mol} / \mathrm{g} ; \Delta p$ is the pressure drop in $\mathrm{mmHg} ; V$ is the volume in $\mathrm{mm}^{3}$. Pressure measurements and recordings during the adsorbentadsorbent coexposure were remotely made while irradiating samples in the central channel of the gamma unit. The error in the pressure measurement was $\sim 5 \%$.

The method of infrared (IR) spectroscopy is one of the most effective methods for the investigation of solid surfaces. This method is widely used as a solution of research and many other problems in Physics, Chemistry and other sciences [20-22]. An infrared spectrometer (KarlZeiss Jena type UR-20) was used with an operating frequency range of $5000-400 \mathrm{~cm}^{-1}$. To reduce IR scattering, beryllium oxide powder particles were repeatedly sieved and pressed into thin pellets at a pressure of $8-12 \mathrm{~kg} / \mathrm{mm}$. Dimensions of the tablets pressed in a special collapsible mould were $30 \times 10 \mathrm{~mm}^{2}$ or $35 \times 15 \mathrm{~mm}^{2}$, depending on irradiation and examination of the samples. The beryllium oxide tablets were $0.2 \mathrm{~mm}$ thick. In case of significant light scattering in the short wavelength range, an aperture was placed in the comparison beam.

Radiation defects in beryllium oxide irradiated by gamma-rays depending on irradiation dose rate and pretreatment temperature were investigated using an electron paramagnetic resonance (EPR) method widely used to study free radicals in various paramagnetic centers formed in the material during the action of ionizing radiation [23, 24]. Before irradiation, beryllium oxide was subjected to several hours of thermal-vacuum treatment. For one series of experiments, the samples were soldered at a pressure $\sim 10^{-6}$ $\mathrm{mmHg}$, and in experiments on co-irradiation of samples with gas, the pressure in ampoules was $10^{-2} \mathrm{mmHg}$. After that, the samples were irradiated either in dewars with liquid nitrogen or at room temperature.

The adsorption and EPR spectra were studied in the same ampoules made of molybdenum glass, giving an intense EPR signal when irradiated. Before the measurements, the ampoules were annealed in a soft flame of a gas burner, and the sample was transferred to the other end of the ampoule and kept either in liquid nitrogen or at room temperature. Before irradiation, the $\mathrm{BeO}$ samples were transferred back into the annealed end of the ampoule. The annealing operation is superfluous when working with Luch glass ampoules, as it is practically not activated under radiation. In this work, radio spectrometers of centimeter $(\lambda=3.2 \mathrm{~cm})$ and decimeter ranges $(\lambda=30 \mathrm{~cm})$ were used to capture EPR spectra. The sensitivity of the spectrometer is 1013 unpaired spins in the sample by diphenylpicrylhydrosyl (DPPH). The resolution of the device was 0.2 Oe. The width of the EPR position spectrum lines and the value of the splitting between the components of the superfine structure were calculated 
from the chart tape of the recorder. The EPR spectra were recorded at room temperature or liquid nitrogen temperature at constant microwave power and instrument sensitivity. DPPH and $\mathrm{Mn}^{+2}$ samples in magnesium oxide, whose positions relative to the sample are strictly fixed, and $\mathrm{CuCl}_{2} \cdot 5 \mathrm{H}_{2} \mathrm{O}$ containing a known amount of paramagnetic particles were used as reference sources in these studies. The g-factors of irradiated $\mathrm{BeO}$ were calculated by the standard method. The reference sources were located either in the dewar or directly in the resonator of the spectrometer.

Before irradiation, samples were annealed for 6-8 $\mathrm{h}$ in air; the same amounts were annealed in a vacuum at $400{ }^{\circ} \mathrm{C}$. Irradiation with ${ }^{60} \mathrm{Co} \gamma$-rays was carried out at dose rate $J=14.4 \mathrm{R} / \mathrm{s}$. Experiments on radiation adsorption kinetics were done at an initial pressure of oxygen and hydrogen of $R_{0}=8.510^{-1}-5.10^{-2}$ torr. IR spectroscopic studies were carried out in the absorption spectrum range of $v=3000-4000 \mathrm{~cm}^{-1}$. EPR studies were performed in both centimeter and decimeter ranges at both room temperature and liquid nitrogen temperature.

\section{Results and discussion}

Above we described the experiments carried out in this research. Detailed studies of the radiation adsorption regularities of hydrogen and oxygen were presented on $\mathrm{BeO}$ samples trained at $473-1023 \mathrm{~K}$ in a radiation field. Fig. 1 demonstrates IR absorption spectra of irradiated $\mathrm{BeO}$ obtained during the experiments.

The inlet of $\mathrm{O}_{2}$ and exposure to $\mathrm{BeO}$ at $673-773 \mathrm{~K}$ decreased the intensity of absorption bands of isolated $\mathrm{OH}$-groups of type $1\left(3750 \mathrm{~cm}^{-1}\right)$, resulting in the appearance of weak peaks of $3680-3720 \mathrm{~cm}^{-1}$ and constant growth of the band of isolated $\mathrm{OH}$-groups $\left(3630 \mathrm{~cm}^{-1}\right)$.

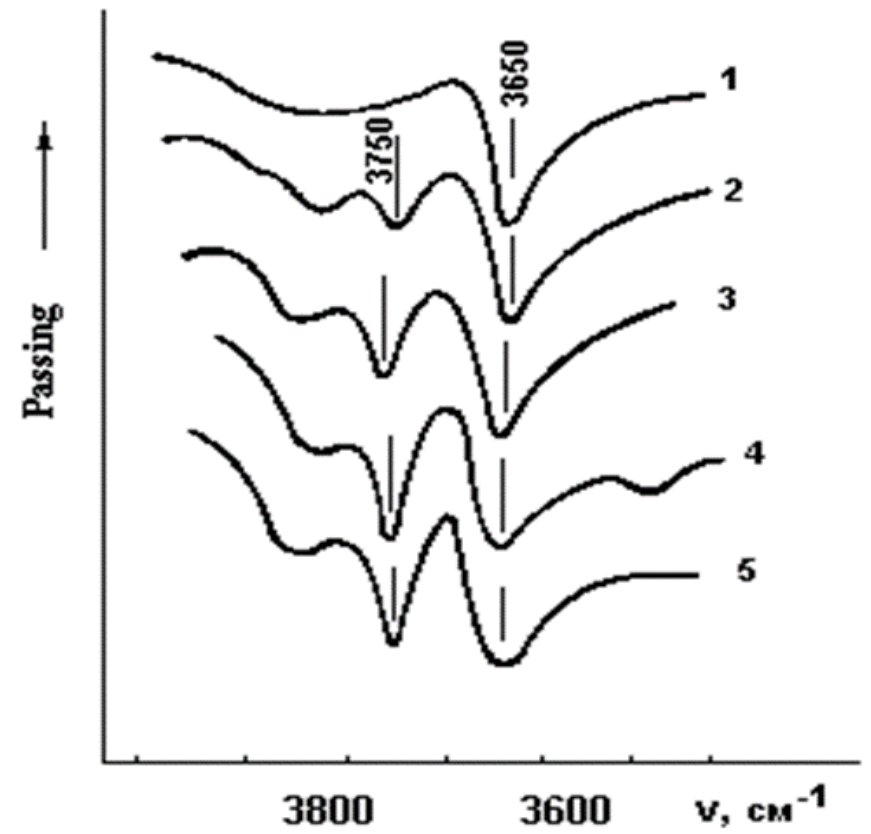

Fig. 1 IR absorption spectra of $\gamma$-irradiated BeO: 1 - initial spectrum before irradiation; 2 - spectrum after irradiation, $D_{\gamma}=0.024 \mathrm{MGy} ; 3$ - puffing of $\mathrm{H}_{2} ; 4$ - in $2.5 \mathrm{~h}$ after puffing; 5 - in $5 \mathrm{~h}$ after puffing with $T_{\mathrm{pr}}=873 \mathrm{~K}$
It was assumed that the decrease in the intensity of the $3750 \mathrm{~cm}^{-1}$ band and the appearance of peaks at $3680-3720 \mathrm{~cm}^{-1}$ are due to the participation of type 1 $\mathrm{OH}$-groups, which play the role of protons in the hydrogen bonding in the oxygen adsorption. In the IR spectrum, along with a decrease in the intensity of hydroxyl groups (primarily the $3740 \mathrm{~cm}^{-1}$ band), bands at 3580 and $3680 \mathrm{~cm}^{-1}$ appear, corresponding to adsorbed water and hydrogen-bonded $\mathrm{OH}$ groups (Fig. 1).

As can be seen from Fig. 2, due to preliminary annealing of samples at $1073 \mathrm{~K}$, oxygen puffing did not cause any changes in the infrared spectrum of $\mathrm{BeO}$, suggesting sintered surfaces.

Fig. 2 represents dose dependence of radiation adsorption of gases on beryllium oxide. This figure shows that the amount of adsorbed gas changed symbatically with the initial pressure and radiation dose while the initial adsorption rate of hydrogen was greater than oxygen. However, at the same initial pressure, for hydrogen adsorption, lower doses were required for saturation compared with oxygen.

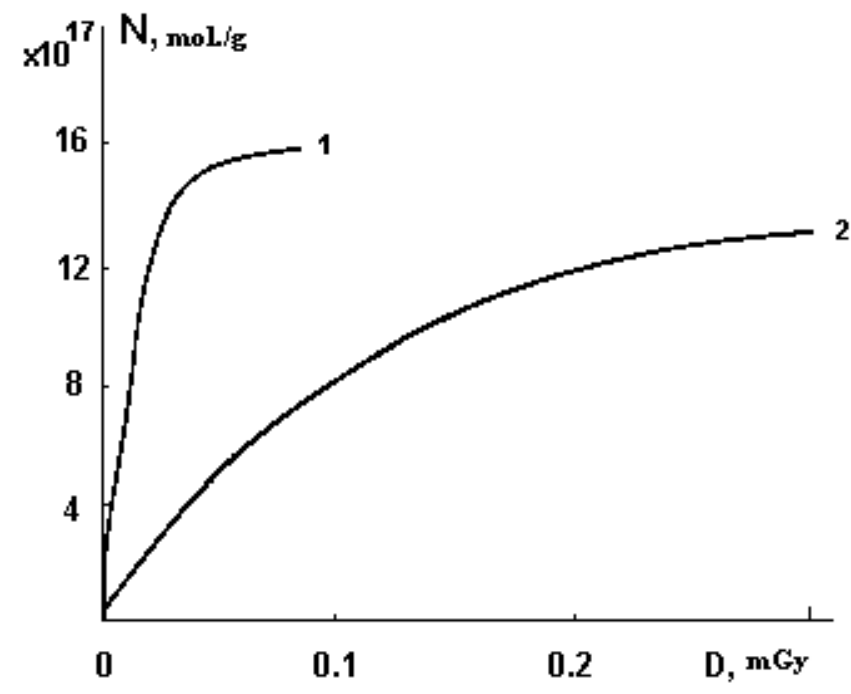

Fig. 2 Dose dependence of radiation adsorption of gases on beryllium oxide: 1 - hydrogen adsorption, 2 - oxygen adsorption $(P=113 \mathrm{~Pa})$

Fig. 3 presents a graphical representation of the kinetics of radiation adsorption of $\mathrm{O}_{2}$ on the $\mathrm{BeO}$ surface.

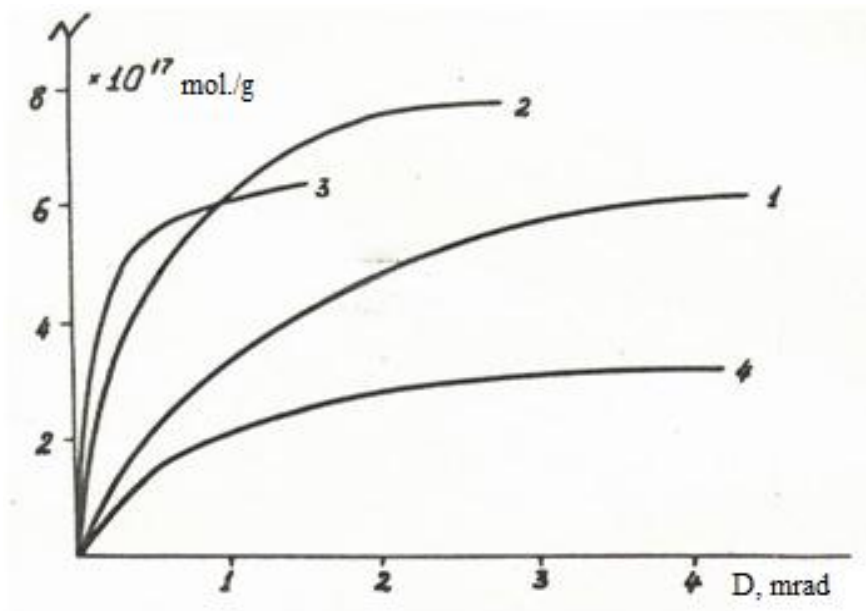

Fig. 3 Kinetics of radiation adsorption of $\mathrm{O}_{2}$ by BeO: $1-P_{0}=1 \cdot 10^{-1}$, $2-1.2 \cdot 10^{-1}, 3-0.5 \cdot 10^{-1}, 4-8.5 \cdot 10^{-1} \mathrm{mmHg}$ 
The result shows that radiation adsorption of oxygen depends on the initial oxygen pressure, i.e., the surface coverage. However, the maximum adsorption activity was observed on samples heated before exposure at $673 \mathrm{~K}$ on $\gamma$-irradiated $\mathrm{BeO}$, as shown in Fig. 4 demonstrating temperature dependence of gamma-adsorption of $\mathrm{O}_{2}$ on beryllium oxide.

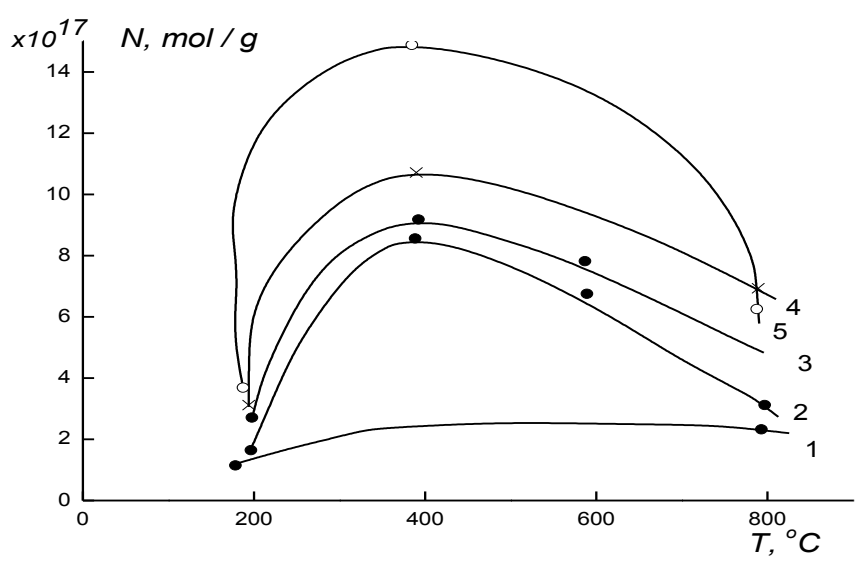

Fig. 4 Distribution in temperature dependence of $\gamma$-adsorption of $\mathrm{O}_{2}$ on $\mathrm{BeO}\left(P_{\mathrm{o}}=9 \cdot 10^{-2} \mathrm{mmHg}\right) D_{\gamma}: 1-0.4 ; 2-1.2 ; 3-2.4 ; 4-4.8$; $5-18.6 \mathrm{mrad}$

The maximum number of paramagnetic centers was also observed at a temperature around $400{ }^{\circ} \mathrm{C}$, i.e., $673 \mathrm{~K}$, as shown in Fig. 5 demonstrating dose dependence of paramagnetic centers on $\mathrm{BeO}$ samples that were calcined before irradiation at different temperatures $\left(T_{\mathrm{c}}\right)$.

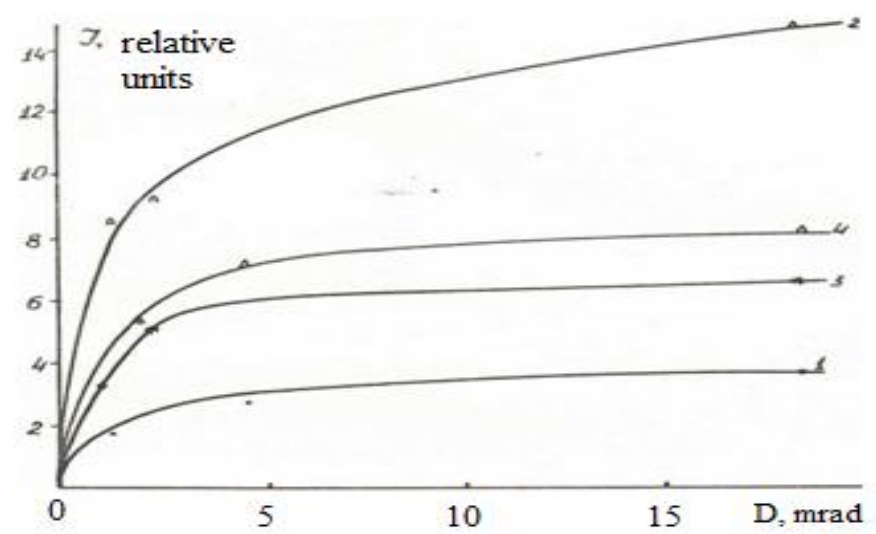

Fig. 5 Dose dependence of paramagnetic centers on BeO: $1-T_{\mathrm{pr}}=200{ }^{\circ} \mathrm{C} ; 2,4-400{ }^{\circ} \mathrm{C} ; 3-600{ }^{\circ} \mathrm{C} ; 4$ - leak-in of $\mathrm{O}_{2}$ $(\lambda=30 \mathrm{~cm})$

Besides, the adsorption of hydrogen caused a decrease in PMC numbers (Fig. 6), indicating adsorption of hydrogen on hole centers and the resulting destruction of such centers. Moreover, during the oxygen adsorption, an increase in the EPR signal is observed, possibly due to the adsorption of oxygen in the paramagnetic form $\mathrm{O}_{2}$.

Thermal desorption of adsorbed $\mathrm{H}_{2}$ and $\mathrm{O}_{2}$ occurred at temperatures $>423 \mathrm{~K}$. Besides, oxygen had two peaks of excitation within 453-533 $\mathrm{K}$ and $573-673 \mathrm{~K}$, corresponding to characteristic ranges of hydrogen-bonded and coordination-bonded molecular release, respectively. Fig. 7 shows dose dependence of PMC in BeO (1) containing adsorbed $\mathrm{O}_{2}$ (2) and adsorbed $\mathrm{H}_{2}$ (3).
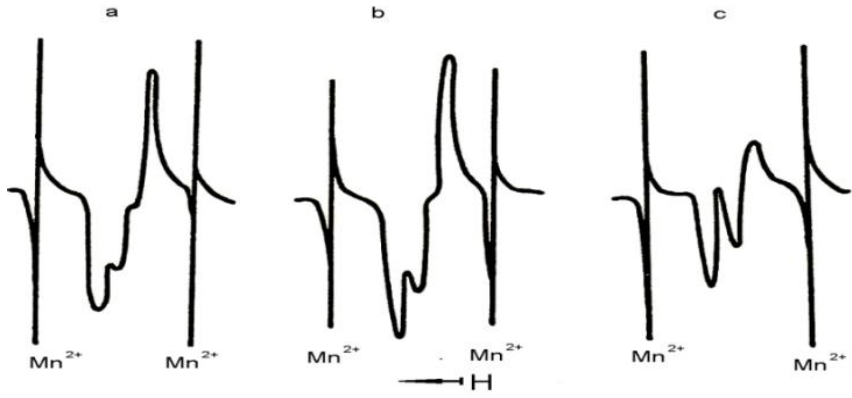

Fig. 6 EPR spectra of gamma-irradiated $\mathrm{BeO}$ (a) with absorbed $\mathrm{O}_{2}$ (b), with adsorbed $\mathrm{H}_{2}\left(D_{\gamma}=60 \mathrm{mrad}\right)(\mathrm{c})$. The irradiation and measurement temperature are ambient

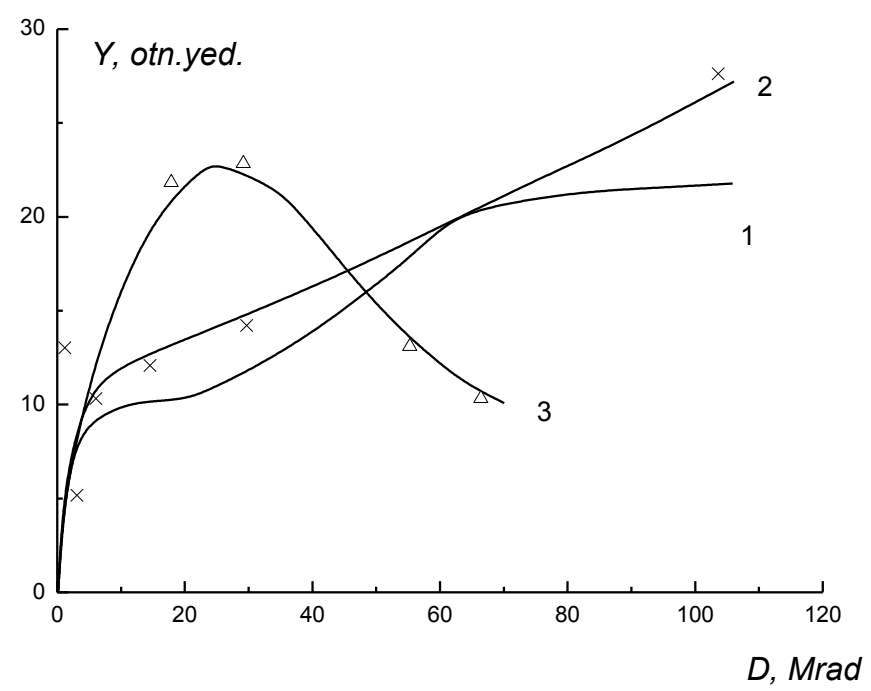

Fig. 7 Dose dependence of PMC on $\mathrm{BeO}$ (1) containing adsorbed $\mathrm{O}_{2}(2)$ and adsorbed $\mathrm{H}_{2}$ (3)

Fig. 8 presents the annealing of paramagnetic centers on $\gamma$-irradiated $\mathrm{BeO}$ (dose of radiation, $D_{\gamma}=18.6 \mathrm{mrad}$ ). The annealing of adsorption and paramagnetic centers ended at different temperatures as the adsorption centers were annealed completely at $673 \mathrm{~K}$, while the PMCs are retained at high temperatures, as described in Fig. 8. It is possible that paramagnetic centers, in contrast to adsorption centers, are also formed in the amount of the sample and, therefore, have higher temperature resistance.

A comparison of infrared spectroscopy, EPR, and manometric data indicated that the $\gamma$-adsorption centers for oxygen and hydrogen are paramagnetic centers with electron and hole regimes. Thus, secondary processes involving surface hydroxyl groups and adsorbed molecules are possible. Further, the possibility of oxidation of organics in the presence of organic matter can not be excluded in this research.

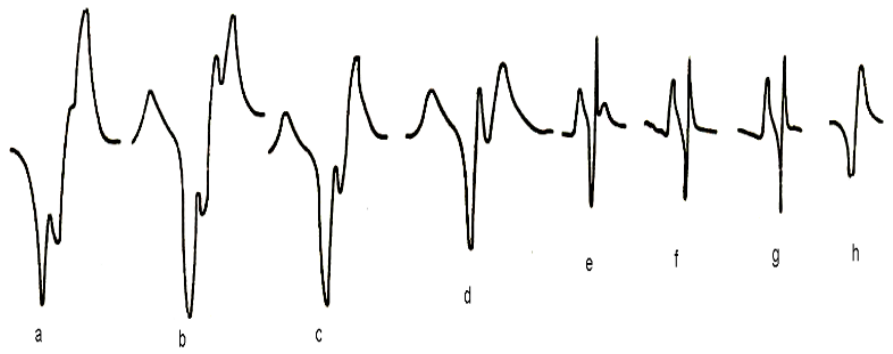

Fig. 8 Annealing of paramagnetic centers on gamma-irradiated BeO. ( $D_{\mathrm{n}}=18.6$ mrad). a $-T=20, \mathrm{~b}-60, \mathrm{c}-120, \mathrm{~d}-170$, e $-240, f-360, g-460,3-480{ }^{\circ} \mathrm{C}$ 


\section{Conclusions}

In summary, a comparison of electron paramagnetic resonance and adsorption studies indicates that absorption of $\mathrm{H}_{2}$ and $\mathrm{O}_{2}$ leads to the destruction of paramagnetic centers, which provides the evidence of formation of adsorption centers with electron and hole regimes on the BeO surface. At the same time, the discrepancy between the absorption dose ranges (up to $0.3 \mathrm{mGy}$ for adsorption and $0.6 \mathrm{mGy}$ and more for EPR centers) and the annealing temperature (573-673 K and below are adsorption centers, $723 \mathrm{~K}$ and above are paramagnetic centers) indicates that these types of centers are not identical. It can be assumed that both surface and bulk paramagnetic centers are formed upon irradiation. The present study will be useful for future research involving absorption kinetics at other metal-oxide surfaces.

\section{Declaration of competing interests}

The authors declare that they have no known competing financial interests or personal relationships that could have appeared to influence the work reported in this paper.

\section{References}

1. Kiyko VS, Vaispapir VY. Production features of preparation and properties of ceramic objects made from a mixture of lightlyand highly-fired $\mathrm{BeO}$ powder. Refract Ind Ceram. 2016;57(4):423-426. doi:10.1007/s11148-016-9997-3.

2. Gaft M, Nagli L, Gorychev A, et.al. Atomic and molecular emission of beryllium by LIBS. Spectrochim Acta Part B: Atomic Spectrosc. 2021;182:106233.

doi:10.1016/j.sab.2021.106233

3. Wroblewska M, Blanchet D, Lyoussi A, et.al. A review and analysis of the state of the art on beryllium poisoning in research reactors. Ann Nucl Energy. 2021;163:108540. doi: $10.1016 /$ j.anucene.2021.108540

4. Zaripov RR, Nazarov AA, Ponikarov SI. Catalysts applied in the catalytic dehydrogenation of hydrocarbons. Bull Kazan Technol Univ. 2013;16:67-75. Available from: https://cyberleninka.ru/article/n/katalizatoryprimenyaemye-pri-kataliticheskom-degidrirovaniiparafinovyh-olefinovyh-i-alkilaromaticheskih-uglevodorodov

5. Dey S, Drazin J, Wang Y, et.al. Radiation tolerance of nanocrystalline ceramics: insights from yttria stabilized zirconia. Sci Rep. 2013;7746. doi:10.1038/srepo 7746

6. Du JH, Peng L. Recent progress in investigations of surface structure and properties of solid oxide materials with nuclear magnetic resonance spectroscopy. Chin Chem Lett. 2018;29(6):747-751. doi:10.1016/j.cclet.2018.02.012

7. Was GS. Fundamentals of radiation materials science. USA, New York: Springer. 2017. doi:10.1007/978-1-4939-3438-6

8. Petrik NG, Alexandrov AB, Orlando TM, Vall AI. Radiationinduced processes at oxide surfaces and interfaces relevant to spent nuclear fuel storage. Am Nucl Soc Winter Meet, Long Beach, CA, United States. 1999.
9. Zinkle SJ, Was GS. Materials challenges in nuclear energy. Acta Materialia. 2013;61(3):735-758. doi:10.1016/j.actamat.2012.11.004

10. Rollett D, Wang Y, Carpenter JS. Design of radiation tolerant materials via interface engineering. Adv. Mater. 2013;25:6975-6979.

11. Polushin NI, Laptev AI, Spitsyn BV. Deposition of borondoped thin CVD diamond films from methane-triethyl boratehydrogen gas mixture. Process. 2020;8(6):666. doi:10.3390/PR8060666

12. Tuseyev T, Polyakov A, Bitenbaev M. About kinetics of detect accumulation in irradiated oxides of metals. Eurasia Conf Nuclear Sci Appl, Izmir Turkey. 2000.

13. Tuseev T. Study of the surface properties of irradiated oxide materials. 6th Int Conf "Nuclear and Radiation Physics", Almaty, Republic of Kazakhstan. 2016.

14. Tuseyev T. Radiation effects on oxide material surface. 5 th Conf "Nuclear Science and Its Application", Ankara, Turkey. 2008.

15. Altunal V, Guckan V, Ozdemir A, et.al. A calcination study on $\mathrm{BeO}$ ceramics for radiation dosimetry. Mater Res Bull. 2020;130. doi:10.1016/j.materresbull.2020.110921

16. Ryskulov AE, Kozlovskiy AL, Ivanov IA, et.al. The effect of $\mathrm{Ni}^{2+}$ heavy ion irradiation on the optical and structural properties of $\mathrm{BeO}$ ceramics. Ceram Int. 2020;46(4):40654070. doi:10.1016/j.ceramint.2019.02.121

17. Khromushin IV, Aksenova TI, Tuseev T, et.al. Particularities of radiation defect formation in ceramic barium cerate. IOP Conf Ser: Mater Sci Eng. 2015;81(1):012037. doi: $10.1088 / 1757-899 \mathrm{X} / 81 / 1 / 012037$

18. Védrine JC. Heterogeneous catalysis on metal oxides. Catal. 2017:7:341. doi:10.3390/catal7110341

19. Mishra SK, Ahlawat A, Khosla D, et.al. Experimental investigation of variations in morphology, composition and mixingstate of boundary layer aerosol: A balloon based study over urban environment (New Delhi). Atmos Environ. 2018;185:243-252. doi:10.1016/j.atmosenv.2018.04.053

20. Martynova TV, Kirichenko AN, Shitareva MS. Fouriertransform infrared spectroscopy for analysis of diamond materials of different origin. IOP Conf Ser: Mater Sci Eng. 2021;1047(1):012187. doi:10.1088/1757-899X/1047/1/012187

21. Altunal V, Guckan V, Ozdemir A, et.al. A systematic study on luminescence characterization of lanthanide-doped $\mathrm{BeO}$ ceramic dosimeters. J Alloys Compd. 2021;876:160105. doi:10.1016/j.jallcom.2021.160105

22. Maslov AL, Laptev AI, Polushin NI. Express-analysis of nitrogen content in CVD-diamonds by FTIR-spectrometry. IOP Conf Ser: Mater Sci Eng. 2020;919(2):022049. doi:10.1088/1757-899X/919/2/022049

23. Milisch B, Kerbe F, Michalowsky L. Defect centres in beryllium oxide ceramics studied by electron paramagnetic resonance (EPR). Ceram Int. 2016;16(5):311-318. doi:10.1016/0272-8842(90)90045-H

24. Fang W, Yang DX, Chen HJ, et.al. Investigations of the electron paramagnetic resonance parameters and defect structures for $\mathrm{Cu}^{2+}$ ions in $\mathrm{BeO}$ crystal with trigonally distorted tetrahedral symmetry. Mater. 2019;475:234-239. doi:10.1016/j.jmmm.2018.11.081 